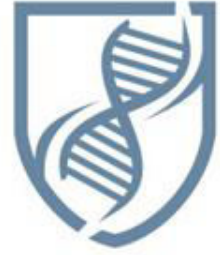

Journal of Bioscience and Applied Research

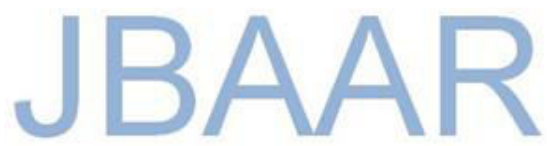

WWW.JBAAR.ORG

\title{
Hepatitis Delta Virus among Hepatitis B Virus Infected Patients in Jordan: Where Do We Stand?
}

\author{
'Samer F. Swedan, ${ }^{2}$ Ziad A. Elnasser and 'Majd N. Brik Hnidi \\ 'Department of Medical Laboratory Sciences, Faculty of Applied Medical Sciences, \\ department of Pathology and Microbiology, Faculty of Medicine, \\ Jordan University of Science and Technology, Irbid, Jordan
}

\begin{abstract}
Infection by Hepatitis Delta Virus (HDV) which can only replicate in the presence of Hepatitis B Virus (HBV) is a cause of significant morbidity and mortality worldwide. Prevalence of HDV in Jordan is lacking. Blood samples were randomly obtained from 100 patients seropositive for active HBV. Sera were analyzed using highly sensitive and specific ELISA kits for the presence of specific HBV and HDV antigens and antibodies. Overall, samples were collected from 70 males and 30 females with an average age of 37.9 years and an age range of 13-62 years. The $94 \%$ of samples were from individuals older than 18 years. All samples were confirmed for the presence of active HBV infection. The 1 and $3 \%$ of the HBV infected individuals demonstrated markers of active and recovered HDV infection, respectively. These findings suggest that HBV positive individuals have a small but significant risk of acquiring HDV. We recommend the implementation of periodical testing for HDV infection among all HBV infected individuals and the administration of the HBV vaccine to unvaccinated individuals.
\end{abstract}

Key words: ELISA, HDV, HBV, hepatitis, infection, prevalence, Jordan

\section{INTRODUCTION}

Hepatitis D disease is caused by Hepatitis Delta Virus (HDV), the most unique and virulent of all hepatitis viruses. HDV was first described in 1977 and is a small, single-stranded, incomplete, enveloped RNA virus that requires the helper functions of $\mathrm{HBV}$ for replication, virion assembly and for penetration and infection of hepatocytes (Bonino et al., 1990; Taylor, 1992; Hadziyannis, 1997; Ogata, 2004). Consequently, HDV is only found in individuals having an active $\mathrm{HBV}$ infection. Infection with HDV may result in either acute or chronic hepatitis (Hoonagle, 1989).

Individuals could be co-infected with HBV and HDV at the same time, usually leading to the development of severe acute disease with low risk of chronic infection. Alternatively, individuals with ongoing $\mathrm{HBV}$ infection could be super-infected with HDV leading to high-risk of severe chronic liver disease (Rizzetto et at, 1991). Individuals infected with both HBV and HDV have high risk of development of fulminant hepatitis, cirrhosis and liver cancer than those having $\mathrm{HBV}$ infection alone
(Rizzetto etal, 1980; Hughes etal, 2011).

HDV shares similar epidemiology and routes of transmission to that of HBV due to codependency on

HBV for replication (Alavian and Alavian, 2005). Worldwide, there are approximately 350 million carriers of HBV, 18 million (i. e., 5\%) of which are infected with HDV (Duarte et at, 2010). Due to the success of HBV vaccination and health care programs, HDV prevalence has been decreasing over time. According to the World Health Organization (WHO), HBV infection is common in the Middle East and HDV can occasionally be found endemically in this area (Toukan et at, 1987; Guneid et at, 1993; Toukan, 1992; Makhmudov et at, 1996; Alavian and Alavian, 2005). HDV infection among asymptomatic carriers of Hepatitis B virus surface antigen ( $\mathrm{HbsAg}$ ) was reported in 2, 31, 3.3 and 5.2\% of cases from Jordan (1987 study) (Toukan et at, 1987), Kuwait (Al-Kandari et at, 1988), Saudi Arabia (Al-Traif et at, 2004) and Turkey (Makhmudov et at, 1996), respectively.

According to the WHO, Jordan is in an area highly endemic for HBV infection with a rate of infection reaching 
up to $10 \%$ (Qirbi and Hall, 2001). This in turn increases risk of infection with HDV and associated sequelae. Information on HDV infection in Jordan is very scarce as

current cross-sectional study was the first to be conducted in Jordan since 1987 to examine the prevalence of HDV in patients seropositive for active HBV through the use of highly sensitive and specific ELISA kits to detect HBV and HDV antigens and antibodies. Information obtained will be crucial as it may require health agencies to reconsider hepatitis screening protocols for donated blood and will help provide information to better define how HDV prevalence varies worldwide.

\section{MATERIALS AND METHODS}

Study samples: The study has been approved by the institutional review board committee. Blood samples demonstrating active HBV infection (HBsAg-positive by ELISA) from 100 Jordanian individuals were randomly obtained from three institutions in the North of Jordan; King Abdullah University Hospital Serology Laboratory, Princess Basma Teaching Hospital Serology Laboratory, and the National Blood Bank in Irbid. Samples were collected between August 2012 and March 2013. Sample inclusion criterion consisted of HBV positive blood with disregard to gender or age. Patients' age and gender were obtained from medical records. Patents' consent was not required since the study was performed on blood samples post-collection and no interactions with patients were required. Serum was obtained from blood samples collected into plain tubes. Serum was separated into several aliquots and stored at $-20^{\circ} \mathrm{C}$ until day of analysis.

Enzyme-Linked Immunosorbent Assay (ELISA): Highly sensitive and specific ELISA kits from Diagnostic automation, Inc. (Calabasas, CA, United States) were used on serum samples to confirm the presence of HbsAg (Cat\# 1701-12) and to identify the presence of anti-HBV surface antigen antibodies (anti-HBs antibodies) (Cat\# 1702-12), anti-HBV core antigen IgM antibodies (anti-HBc IgM antibodies) (Cat\# 1778-12), anti-HBc total IgM and IgG antibodies (Cat\# 1704-12), HDV delta antigen (Cat\# 186812) and anti-HD V delta antigen IgG antibodies (Cat\# 1870-12). All tests were performed according to only one study exists that provides light on this issue (Toukan et at, 1987). However, this study was done in 1987 and does not presently apply. Therefore, the

manufacture instructions and included the appropriate positive and negative controls and standard concentration curves.

Statistics: The statistical package for the social sciences (SPSS, IBM Inc., Version 21) was used for data analysis and for generation of all cross-tabulations. Pearson's $\mathrm{y}^{2}$-test was used to identify significance of frequency differences. A $p<0.05$ was considered significant.

\section{RESULTS AND DISCUSSION}

Blood samples from 100 patients seropositive for HBV were obtained randomly from 3 medical institutions from northern Jordan. The samples were obtained from 70 males and 30 females. The age of patients ranged from 1362 years with a mean of 37.9 years and a standard deviation of 11.8 years. The median age was 38 years. Age groups (listed in decreasing frequency) 30-39, 40-49, 20-29 and 50-59 years, accounted for the majority of samples (Table $1)$.

ELISA results according to patients' age groups are demonstrated in Table 1. All 100 samples were positive for HBsAg and 94 samples (94\%) were negative for anti-HBs antibodies. Only one sample was positive for anti-HBc IgM antibody. All samples were positive for total (IgM and $\mathrm{IgG}$ ) anti-HBc antibodies. Regarding HDV infection, only one sample $(1 \%)$ was positive for the HDV delta antigen. This sample belonged to a female 55 years of age. Three samples $(3 \%)$ were positive for anti-HDV IgG antibodies. The samples belonged to a 13 years old male, a 38 years old male and a 38 years old female.

HDV infection can only occur with HBV infection due to co-dependency on HBV for replication. This infection is associated with high medical cost and high rates of morbidity and mortality. Furthermore, many infected individuals go undiagnosed and pose a risk for further transmission within the population. Thus, prevalence studies are essential to characterize its presence, in order to facilitate application of control measures to minimize its impact on the population.

Table 1: Distribution of patient gender and ELISA results according to age groups

\begin{tabular}{|c|c|c|c|c|c|c|c|c|c|c|c|c|c|c|c|}
\hline \multirow{2}{*}{$\begin{array}{l}\text { Patient age } \\
\text { group } \\
\text { (years') }\end{array}$} & \multicolumn{2}{|l|}{ Gender } & \multicolumn{2}{|c|}{ HbsAg } & \multicolumn{2}{|c|}{ Anti-HBs Ab } & \multicolumn{2}{|c|}{$\begin{array}{l}\text { Anti-HBc } \\
\text { IgM Ab }\end{array}$} & \multicolumn{2}{|c|}{$\begin{array}{l}\text { Total anti- } \mathrm{HBc} \\
\mathrm{Ab}\end{array}$} & \multirow{2}{*}{$\begin{array}{l}\text { HD } \\
\mathrm{V} \\
\text { delta }\end{array}$} & \multicolumn{3}{|c|}{$\begin{array}{l}\text { Anti-HDV } \\
\text { IgG Ab }\end{array}$} & \multirow[b]{2}{*}{ Total } \\
\hline & $\mathrm{F}$ & $\mathrm{M}$ & & + & & + & & + & & + & & + & & + & \\
\hline $10-19$ & 1 & 5 & 0 & 6 & 5 & 1 & 6 & 0 & 0 & 6 & 6 & 0 & 5 & 1 & 6 \\
\hline $20-29$ & 9 & 9 & 0 & 18 & 17 & 1 & 18 & 0 & 0 & 18 & 18 & 0 & 18 & 0 & 18 \\
\hline $30-39$ & 12 & 24 & 0 & 36 & 35 & 1 & 35 & 1 & 0 & 36 & 36 & 0 & 34 & 2 & 36 \\
\hline $40-49$ & 4 & 17 & 0 & 21 & 19 & 2 & 21 & 0 & 0 & 21 & 21 & 0 & 21 & 0 & 21 \\
\hline $50-59$ & 4 & 14 & 0 & 18 & 17 & 1 & 18 & 0 & 0 & 18 & 17 & 1 & 18 & 0 & 18 \\
\hline $60-69$ & 0 & 1 & 0 & 1 & 1 & 0 & 1 & 0 & 0 & 1 & 1 & 0 & 1 & 0 & 1 \\
\hline Total & 30 & 70 & 0 & 100 & 94 & 6 & 99 & 1 & 0 & 100 & 99 & 1 & 97 & 3 & 100 \\
\hline
\end{tabular}

F: Female; M: Male; HBsAg: HBV surface antigen; Anti-HBs Ab: antibody against HBV surface antigen; Anti-HBc IgM Ab: IgM antibody against HBV core antigen. Total anti-HBc Ab: Total IgM and IgG antibodies against HBV core antigen. HDV delta Ag: HDV delta antigen. Anti-HDV IgG Ab: IgG antibody against HDV delta antigen; negative ELISA test and "+": positive ELISA test 
To our knowledge, this is the first Jordanian HDV study since 1987 (Toukan et al., 1987), to investigate the prevalence of HDV among patients seropositive for active HBV infection. Overall, the HBV positive samples were obtained from 70 males and 30 females $(p<0.001)$. This bias of higher frequency of HBV positive samples from males could be explained by the fact that 45 samples were from blood donors attending blood bank and that the majority of blood donors in Jordan are males. All 100 samples were positive for HBsAg. This confirms the serological diagnosis initially performed at the institution at which the samples were obtained. This also indicates that all samples included in the study were from individuals having active HBV infection.

The samples belonged to patients with an age range of 13-62 years. However, 94\% of samples were obtained from patients older than 18 years, suggesting that young individuals were less likely to be infected with HBV. Interestingly, Jordan has introduced routine $\mathrm{HBV}$ vaccination in 1995 , indicating that at the time the study samples were collected, most Jordanians < 18 years of age should have been vaccinated. This might explain the low frequency of infection among the 10-19 years age group. Alternatively, individuals older than 18 years who are less likely to have been vaccinated, were at higher risk of acquiring HBV infection due to blood transfusions, sexual activities and other risk factors. This finding highlights the importance of vaccination in prevention of HBV infection and consequently prevention of HDV infection in the population.

Antibodies to $\mathrm{HBc}$ antigen are detectable in serum or plasma shortly before clinical symptoms and shortly after the appearance of $\mathrm{HBsAg}$. After resolution of $\mathrm{HBV}$ infection, anti-HBc antibodies appear in blood during the window period following loss of $\mathrm{HBsAg}$ and prior to the development of antibody to $\mathrm{HBsAg}$. Anti-HBc antibodies are found in acute and chronic hepatitis B patients. Therefore, detection of anti-HBc antibodies could be used as a marker for $\mathrm{HBV}$ infection. In this study all 100 samples were positive for total $\operatorname{IgM}$ and $\operatorname{IgG}$ antibodies against $\mathrm{HBc}$ antigen. However, only one sample was positive for the IgM antibody alone. This suggests that only one patient had been recently infected by HBV while the remaining patients had an older infection.

During HBV-HDV co-infection which usually results in acute disease, detectable concentrations of HDV specific antigens first appear 6-8 weeks after exposure. During convalescence, antibodies against HDV antigens appear and are associated with clearance of the antigens. Therefore, detection of circulating HDV delta antigens indicates current infection (Alavian and Alavian, 2005). During HDV super-infection, detectable levels of HDV delta antigens appear 2 weeks following exposure. The failure to clear these antigens indicates possible progression to long term, chronic carrier stage (Alavian and Alavian, 2005). In this study, we used an ELISA kit having 100\% sensitivity and specificity and that could detect extremely low levels of HDV delta antigens. Using this kit, only one sample, belonging to a 55 years old female, had a positive result indicating current $\mathrm{HDV}$ infection. However, 3 patients who were negative for the delta antigen demonstrated positive anti-HDV antibodies indicating previously resolved HDV infections. Real-time PCR could be used for the detection of active HDV infections and is associated with high sensitivity and specificity. However, it is not available for routine diagnosis at clinical laboratories (Negro and Rizzetto, 1994; Huang and Lo, 2010). Therefore, we opted for ELISA detection of HDV delta antigen as an indicator of active HDV infection as we wanted to assess its utility as a potential future routine screening test.

The serological detection of anti-HBs antibodies is used as an indicator for successful vaccination and protection after natural $\mathrm{HBV}$ infection and for monitoring of HBV disease progression. In this study, this test was included to determine if patients had recovered from $\mathrm{HBV}$ infection. The anti-HBs antibody is the last antibody to appear in serum after the resolution of infection. However, 6 out the 100 samples that were positive of HBsAg were also positive for anti-HBs antibodies. This suggests that the individuals were at the early convalescence stage of HBV disease. The ELISA kits used had a specificity of $99.87 \%$ for HBsAg and $99.70 \%$ for anti-HBs antibodies, thus, making the possibility of false positive results due to ELISA non-specificity unlikely. Interestingly, one out of the six individuals, a 13 years old male was also positive for anti-HDV antibody and negative for HDV delta antigen, suggesting that he had recovered from a previous HDV infection and is currently recovering from an HBV infection.

The prevalence of active HDV infection among Jordanians seropositive for HBV in 2013 is $1 \%$ (this study) while in 1987, among asymptomatic HBsAg carriers it was 2\% (Toukan et al., 1987). Compared to surrounding countries, HDV infection was present at a low rate. In Kuwait (Al-Kandari et al., 1988), Saudi Arabia (Al-Traif et al., 2004) and Turkey (Makhmudov et al., 1996), HDV infection was present in $31,3.3$ and $5.2 \%$, respectively of individuals seropositive for active HBV infection. The apparent small decrease in rate of HDV infection, from 1987-2013, among HBV-infected individuals could be attributed to the unavailability of sensitive detection methods in 1987, the introduction of the HBV vaccination program or current availability of better hepatitis virus infection detection and treatment. 
Based on the findings of this study we recommend the implementation of hepatitis virus awareness programs to educate the general population on the modes of $\mathrm{HBV}$ and HDV transmission and on the consequences of infection. We recommend the implementation of periodical testing to monitor HBV progression and to identify HDV coinfection. This should lead to decreased patient morbidity and mortality. Since most HBV infections occur in the HBV unvaccinated age group (i.e., >18 years), we recommend that a nationwide $\mathrm{HBV}$ vaccination program be implemented to target this population. This would significantly decrease HBV infection incidence and would virtually eliminate the risk of HDV infection. This is consistent with a suggestion by the WHO of an expanded vaccination program throughout the world. Finally, we recommend the expansion of routine hepatitis virus screening in blood banks to include HDV testing. Screening could be performed using ELISA.

\section{CONCLUSION}

In summary, this study reported 1 and $3 \%$ of active and recovered HDV infection rates, respectively, among $\mathrm{HBV}$ infected individuals. Since, the reported rate of HBV infection among the Jordanian population is $2-3 \%$, we therefore, deduce that the rate of active HDV infection overall is approximately $0.02-0.03 \%$.

\section{ACKNOWLEDGEMENT}

This study was funded in full by the research deanship at Jordan University of Science and Technology.

\section{REFERENCES}

Al-Kandari, S., E. Nordenfelt, B. Al-Nakib, B.G. Hansson, K. Ljunggren and W. Al-Nakib, 1988. Hepatitis delta virus infection in acute hepatitis in Kuwait. Scand. J. Infect. Dis., 20: 15-19.

Al-Traif, I.M.D., 2004. Prevalence of hepatitis delta antibody among HBsAG carriers in Saudi Arabia. Ann Saudi Med., 24: 343-344.

Alavian, S.M. and S.H. Alavian, 2005. Hepatitis D virus infection; Iran, Middle East and Central Asia. Hepat Mon, 5: 137-143.

Bonino, F., F. Negro, M.R. Brunetto and G. Verme, 1990. "Hepatitis delta virus infection". Prog Liver Dis. 9: 485-496.

Duarte, M.C., N. Cardona, F. Poblete, K. Gonzalez and M. Garcia et al., 2010. A comparative epidemiological study of hepatitis B and hepatitis D virus infections in Yanomami and Piaroa Amerindians of Amazonas
State, Venezuela. Trop. Med. Int. Health, 15: 924-933.

Guneid, A.M.E., A. A. Gunaid, A.M. O'Neill, N.I. Zureikat and J.C. Coleman etal., 1993. Prevalence of hepatitis $\mathrm{B}, \mathrm{C}$ and $\mathrm{D}$ virus markers in Yemeni patients with chronic liver disease. J. Med. Virol., 40: 330-333.

Hadziyannis, S.J., 1997. Review: Hepatitis delta. J. Gastroenterol. Hepatol., 12: 289-298.

Hoonagle, J.H., 1989. Type D (delta) hepatitis. JAMA, 261: 1321-1325.

Huang, C.R. and S. J. Lo, 2010. Evolution and diversity of the human hepatitis D virus genome. Adv. Bioinf., 2010: 1-9.

Hughes, S.A., H. Wedemeyer and P.M. Harrison, 2011. Hepatitis delta virus. The Lancet, 378: 73-85.

Makhmudov, O.S., F.I. Inoyatova, B.A. Kadirov and S.U. Abdumadjidova, 1996. Delta infection in children with chronic viral hepatitis B. Turk. J. Pediatr., 39: 7580.

Negro, F. and M. Rizzetto, 1994. Diagnosis of hepatitis delta virus infection. J. Hepatol., 22: 136-139.

Ogata, N., 2004. Hepatitis Delta (D) virus infection: general aspects of epidemiology, infection modes and clinical picture. Nihon rinsho. Jpn. J. Clin. Med., 62: 406-411.

Qirbi, N. and A.J. Hall, 2001. Epidemiology of hepatitis B virus infection in the Middle East. Eastern Mediterranean Health J., 7: 1034-1045.

Rizzetto, M., A. Ponzetto and I. Forzani, 1991. Epidemiology of hepatitis delta virus: An overview. Progress Clin. Biol. Res., 364: 1-20.

Rizzetto, M., B. Hoyer, M.G. Canese, J.W. Shih, R.H. Purcell and J.L. Gerin, 1980. Delta agent: Association of delta antigen with hepatitis B surface antigen and RNA in serum of delta-infected chimpanzees. Proc. Nat. Acad. Sci., 77: 6124-6128.

Taylor, J.M., 1992. The structure and replication of hepatitis delta virus. Annu. Rev. Microbiol., 46: 2532760 .

Toukan, A.U., 1992. An overview of hepatitis D virus infection in Africa and the Middle East. Prog. Clin. Biol. Res., 382: 251-258.

Toukan, A.U., O.A. Abu-El-Rub, S.A. Abu-Laban, M.S. Tarawneh and M.F. Kamal et al., 1987. The epidemiology and clinical outcome of hepatitis D virus (delta) infection in Jordan. Hepatol., 7: 13401345 . 